\title{
A novel small inhibitor, LLL12, targets STAT3 in non-small cell lung cancer in vitro and in vivo
}

\author{
YANLI NIE ${ }^{1}$, YUANYUAN $\mathrm{LI}^{2}$ and SHENG HU ${ }^{1}$ \\ ${ }^{1}$ Department of Gastrointestinal Medical Oncology, Hubei Province Cancer Hospital; \\ ${ }^{2}$ College of Chemistry, Central China Normal University, Wuhan, Hubei 430079, P.R. China
}

Received July 13, 2016; Accepted April 16, 2018

DOI: $10.3892 /$ ol.2018.9262

\begin{abstract}
Lung cancer is the leading cause of cancer-associated mortality worldwide. Despite the development of novel molecular therapies, the prognosis for patients with locally advanced or metastatic lung cancer remains poor. Therefore, the identification of novel therapeutic approaches is required. In numerous types of cancer, the constitutive activation of signal transducer and activator of transcription 3 (STAT3) signaling serves as a potent therapeutic target. The present study aimed to characterize the suppressive role of LLL12, a STAT3 small molecule inhibitor, in lung cancer cell proliferation and tumor growth. The mechanism of STAT3 signaling modulation by LLL12 was also investigated. The antitumor activity of LLL12 was revealed to take place via inhibition of lung cancer cell proliferation and migration in vitro. High and low doses of LLL12 significantly reduced tumor volume and weight in xenograft mice compared with that in the control group. Furthermore, LLL12 was demonstrated to reduce the level of STAT3 phosphorylation. These results suggested that LLL12 inhibited the proliferation and migration of A549 cells, and the increase in tumor volume in nude mice with lung cancer. This may be associated with the inhibitory effect of STAT3 phosphorylation and the expression of STAT3. The results of the present study suggest that constitutive STAT3 signaling is required for lung cancer cell survival and migration, and tumor growth in vivo. It is also indicated that LLL12 has clinical potential as a novel targeted therapy.
\end{abstract}

\section{Introduction}

Lung cancer is the most common type of malignancy and the leading cause of cancer-associated mortality worldwide (1). Despite the identification of novel molecular therapies and

Correspondence to: Dr Sheng Hu, Department of Gastrointestinal Medical Oncology, Hubei Province Cancer Hospital, 16 Zhuodaoquan South Road, Hongshan, Wuhan, Hubei 430079, P.R. China

E-mail: ehusmn@163.com

Key words: signal transducer and activator of transcription 3, lung cancer, small molecular inhibitor driver oncogenes (2), the prognosis for patients with locally advanced or metastatic lung cancer remains poor, with an overall 5-year survival rate of $<15 \%$ (3). This highlights the urgent requirement for the identification of novel and effective therapeutic agents.

The Janus kinase 2 (JAK2)/signal transducer and activator of transcription 3 (STAT3) signaling pathway is involved in oncogenesis and cancer development (4). STAT3 belongs to the STAT family of transcription factors, which serve diverse roles in cell proliferation, differentiation, apoptosis and ontogenesis (5). It has been suggested that STAT3 also serves an important role in cancer angiogenesis $(5,6)$. STAT3 expression is dysregulated in various types of hematopoietic malignancies and solid tumors, including leukemia, lymphoma, pancreatic cancer, breast cancer, head and neck cancer, skin cancer, melanoma, brain glioblastoma, prostate cancer, liver cancer and lung cancer $(7,8)$.

Constitutive activation of the STAT3 pathway can inhibit the apoptosis of tumor cells by upregulating anti-apoptotic proteins, including $\mathrm{Bcl} 2$, apoptosis regulator $(\mathrm{Bcl} 2)$, Bcl-extra-large (Bcl-xl), myeloid cell leukemia-1 (Mcl-1) and surviving (9). Other proliferation-associated proteins, including cyclin D1 and myc, and the pro-angiogenic vascular endothelial growth factor (VEGF), are also regulated by the JAK-STAT3 signaling pathway (10). STAT3 and proto-oncogene c-Jun inhibit the expression of Fas cell surface death receptor, which may inhibit cancer cell apoptosis (11). Numerous studies have demonstrated that the inhibition of STAT3 prevents tumor growth and causes the apoptosis of tumor cells $(12,13)$. A number of anti-angiogenesis therapeutic strategies have been suggested to block STAT3 pathway signaling, including upstream kinase inhibition, peptide or small molecule inhibitor phosphorylation, dominant-negative STAT3 mutations and oligonucleotide decoys (14-16). However, these methods have limitations, including poor stability and low transduction rates, and have not performed effectively in clinical trials $(17,18)$. Using structure-based design, Professor Li and his team (Ohio State University, Columbus, Ohio, USA) developed LLL12, a non-peptide small molecule inhibitor of STAT3, which possesses good solubility and predictable oral bioavailability. LLL12 binds to the phosphorylated tyrosine of STAT3 monomers, blocking dimerization and subsequent translocation into the nucleus. This abrogates the function of STAT3 as a transcription factor (19). 
A number of studies have demonstrated that LLL12 has potent antitumor activity in breast cancer, brain cancer, pancreatic cancer and hepatocellular carcinoma (19-21). However, to the best of our knowledge, the activity of LLL12 in lung cancer has not been previously investigated. The aim of the present study was to characterize the biological activity and possible underlying mechanism of LLL12 in lung cancer in vitro and in vivo.

\section{Materials and methods}

Cell lines and reagents. The human lung adenocarcinoma cell line, A549, was purchased from the Type Culture Collection Center of Wuhan University (Wuhan, China). The cells were maintained in RPMI-1640 medium containing 10\% fetal bovine serum (Shanghai Baoman Biotechnology Co., Ltd., Shanghai, China) at $37^{\circ} \mathrm{C}$ in $5 \% \mathrm{CO}_{2}$. The following primary antibodies were used for western blotting experiments: Anti-p-STAT3 mAb (Y705; cat. no. ab76315; 1:500 dilution), anti-STAT3 mAb (cat. no. ab5073; 1:500 dilution), anti-p-Src mAb (cat. no. ab40660; 1:500 dilution), anti-p-ERK1/2 mAb (cat. no. ab176660; 1:500 dilution) and anti-GAPDH mAb (cat. no. ab8245; 1:1,000 dilution) (all Abcam, Cambridge, UK). The secondary antibody was HRP-conjugated anti-mouse IgG (cat. no. ab222759; Abcam). LLL12 was kindly gifted by Professor Tom Li (Ohio State University, Columbus, Ohio, USA).

Mice. Female BALB/c nude mice were purchased from Beijing Vital River Laboratory Animal Co. Ltd. (Beijing, China), with the following production license number: SCXK (Beijing) 2006-0009. A total of 25 mice, 4-6 weeks of age, weighing 18.0-22.0 g, were kept three to five mice per cage in microisolator units and provided with water and food in accordance with Institutional Animal Care and Use Committee (IACUC) guidelines, at a temperature of $26-28^{\circ} \mathrm{C}$ and $40-60 \%$ humidity. The present study was approved by the Ethics Committee of Hubei Cancer Hospital (Wuhan, China).

MTT assay. A549 cells were cultured at $1 \times 10^{3}$ cells $/ \mathrm{ml}$ in 96-well plates with RPMI-1640 medium. Cells were cultured with $0.0,0.625,1.25,2.5,5.0$ or $10.0 \mu \mathrm{M} \mathrm{LLL12}$ at $37^{\circ} \mathrm{C}$ for $24,48,72$ or $96 \mathrm{~h}$. A total of $50 \mu 15 \mathrm{mg} / \mathrm{ml} \mathrm{MTT}$ was added per well. After $4 \mathrm{~h}$, the formazan crystals were dissolved by adding $100 \mu 1$ dimethyl sulfoxide (DMSO). The absorbance was read at $490 \mathrm{~nm}$, and the $\mathrm{IC}_{50}$ values were calculated using SigmaPlot9.0 software (Systat Software, Inc., San Jose, CA, USA). Each experiment was repeated 3 times.

Wound-healing assay. A wound-healing assay was performed, in which $2 \times 10^{4}$ cells/well were cultured in RPMI-1640 supplemented with $10 \%$ fetal calf serum. LLL12 (at the $\mathrm{IC}_{50}$ concentration: $5.0 \mu \mathrm{mol} / \mathrm{l}$ ) DMSO or PBS (blank control) was added to each well and incubated at $37^{\circ} \mathrm{C}$ for $24 \mathrm{~h}$. A P10 pipette tip was used to create a $0.5-\mathrm{cm}$ wound in the cell monolayer. The cells were then cultured in serum-free medium for $24 \mathrm{~h}$ prior to washing in PBS. Images of the cells were captured at 0 and $24 \mathrm{~h}$ under an inverted fluorescence microscope (magnification, x100). All experiments were performed in triplicate.

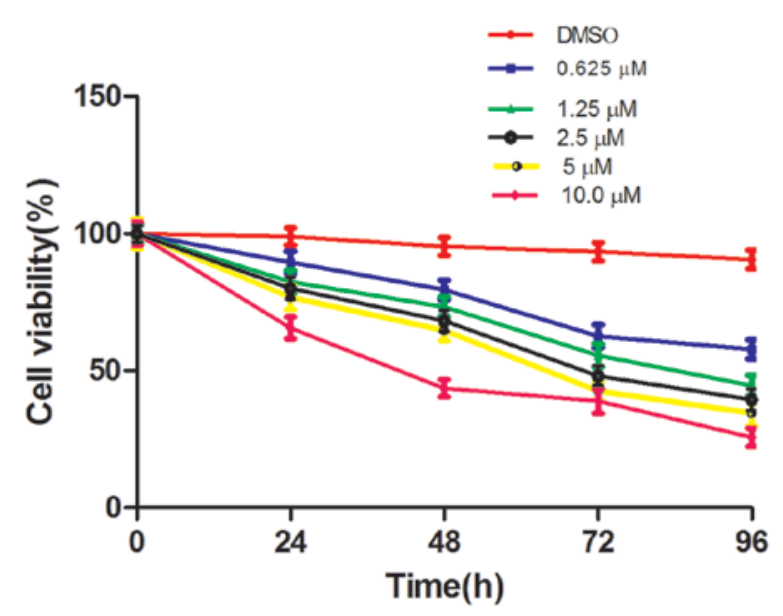

Figure 1. Effect of LLL12 on the proliferation of A549 cells. DMSO, dimethyl sulfoxide.

Migration assays. A Transwell assay was also performed, in which $2 \times 10^{5}$ cells treated with LLL12 (at the $\mathrm{IC}_{50}$ concentration: $5.0 \mu \mathrm{mol} / \mathrm{l}$ ), DMSO or PBS (blank control) in serum-free medium, were seeded into the upper chambers of 6-well plates containing polycarbonate Transwell inserts (pore diameter, $5 \mu \mathrm{m}$; Corning Costar; Corning Incorporated, Corning, NY, USA). Soluble stromal-cell derived factor 1 (PeproTech, Inc., Rocky Hill, NJ, USA) was added at a concentration of $100 \mathrm{ng} / \mathrm{ml}$ to the lower chamber in DMEM medium supplemented with $10 \%$ fetal calf serum. The plates were incubated at $37^{\circ} \mathrm{C}$ for $18 \mathrm{~h}$. Images were captured of the cells that had migrated to the lower surface of the Transwell membrane and then the cells were counted under a light microscope at magnification, $\mathrm{x} 200$. All experiments were performed in triplicate.

Western blot analysis. Cells were treated with DMSO or LLL12 (at the $\mathrm{IC}_{50}$ concentration: $5.0 \mu \mathrm{mol} / \mathrm{l}$ ) for $24 \mathrm{~h}$ prior to protein isolation. The cells were washed twice with cold PBS, then lysed using radioimmunoprecipitation assay buffer $[10 \mathrm{mM}$ Tris (pH 8.0), $150 \mathrm{mM} \mathrm{NaCl}, 1 \%$ sodium deoxycholate, $0.1 \%$ sodium dodecyl sulfate (SDS), $1 \%$ Triton X-100, $10 \mu \mathrm{g} / \mathrm{ml}$ leupeptin, $10 \mu \mathrm{g} / \mathrm{ml}$ aprotinin and $1 \mathrm{mM}$ phenylmethylsulfonyl fluoride]. The cell lysates were centrifuged at 13,000 x g at $4^{\circ} \mathrm{C}$ for $20 \mathrm{~min}$. The protein concentration was determined by bicinchoninic acid protein assay (Thermo Scientific, IL, USA). Protein ( $40 \mu \mathrm{g}$ per lane) was separated by $0.1 \%$ SDS-PAGE, and transferred onto Hybond polyvinylidene difluoride membranes. The membranes were blocked with Tris-buffered saline buffer (20 mM Tris- $\mathrm{HCl}, 150 \mathrm{mM} \mathrm{NaCl}, \mathrm{pH}$ 7.4) containing 5\% non-fat milk for $1 \mathrm{~h}$ at $25^{\circ} \mathrm{C}$, then incubated with the aforementioned primary antibodies for $2 \mathrm{~h}$ at room temperature. Incubation with the horseradish peroxidase-conjugated secondary antibody followed, prior to washing. The membranes were imaged using Scanner STORM 860 (GE Healthcare, Chicago, IL, USA). The relative expression levels of p-Src (Tyr418), p-STAT3 (Y705), STAT3, p-ERK1/2 (T202/Y204) proteins were quantitated using Matrox Inspector software (version 2.1; Matrox Electronic Systems Ltd. Dorval, QC, Canada).

Mouse xenograft model. The 25 mice were injected subcutaneously into the left flank with $5 \times 10^{6}$ A549 cells. Once the 

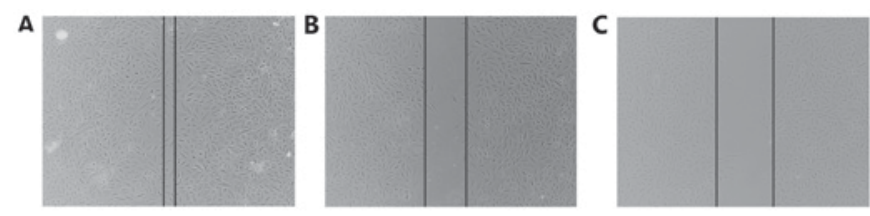

Figure 2. A549 cells treated with LLL12 are less migratory than control cells. Representative photomicrographs of wound healing assays of (A) A549 cells treated with DMSO; (B) blank control A549 cells, and (C) A549 cells treated with LLL12.

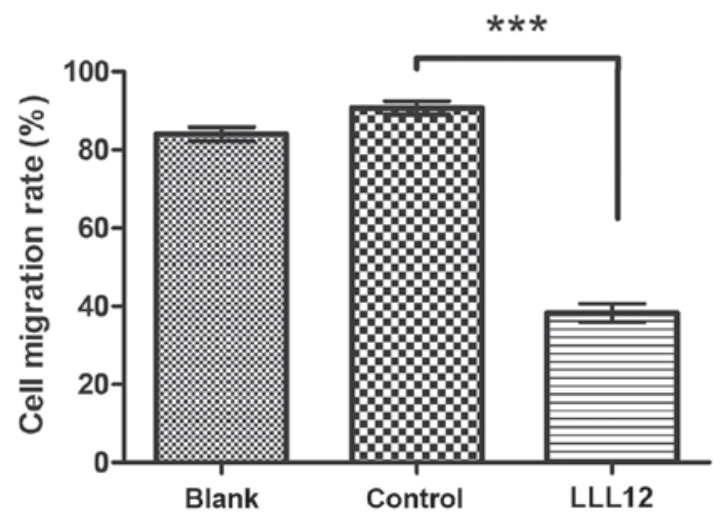

Figure 3. A549 cells treated with LLL12 are less migratory than control cells. The cells were counted under a light microscope. The cell migration rate in the LLL12 treatment group was significantly reduced compared with that in the control group. $\left.{ }^{* * * *} \mathrm{P}<0.001\right)$. and the difference between the blank control and LLL12 group was also significant.

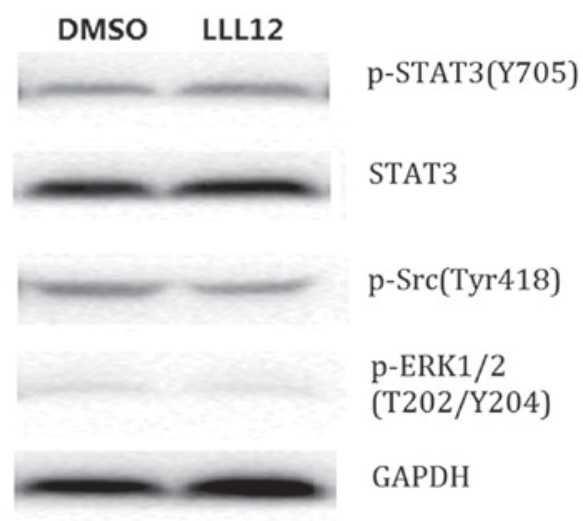

Figure 4. Western blotting analysis of the effect of LLL12 on the protein expression level of members of the JAK-STAT3 pathway. JAK, Janus kinase; STAT3, signal transducer and activator of transcription 3; p-, phosphorylated; Src, proto-oncogene, non-receptor tyrosine kinase; ERK1/2, extracellular signal-regulated kinase 1/2; DMSO, dimethyl sulfoxide.

tumors reached $100-150 \mathrm{~mm}^{3}$ in size, the mice were randomly assigned into the high-dose $(20 \mathrm{mg} / \mathrm{kg}$ LLL12, administered at a concentration of $4 \mathrm{mg} / \mathrm{ml})$, low-dose $(10 \mathrm{mg} / \mathrm{kg}$ LLL12, administered at a concentration of $2 \mathrm{mg} / \mathrm{ml}$ ) or control (administered with an equal volume of DMSO) group. There were 5 mice in each group, and treatment was administered by daily intraperitoneal injection. Tumor growth was determined by caliper measurements of the length $(\mathrm{L})$ and width

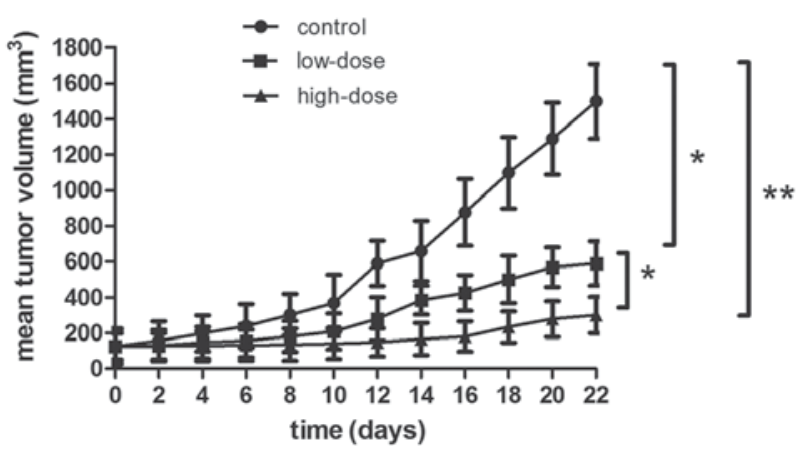

Figure 5. Tumor volumes of mice treated with LLL12 were significantly reduced compared with that of the control group, and those of the high-dose group were significantly reduced compared with those of the low-dose group. ${ }^{*} \mathrm{P}<0.05,{ }^{* *} \mathrm{P}<0.01$.
A

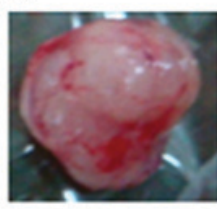

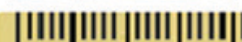

B

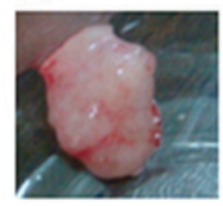

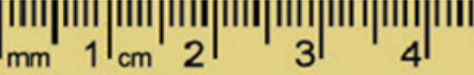

C

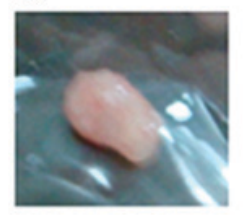

Figure 6. Representative images indicating tumor size. (A) Control group, (B) low-dose group and (C) high-dose group.

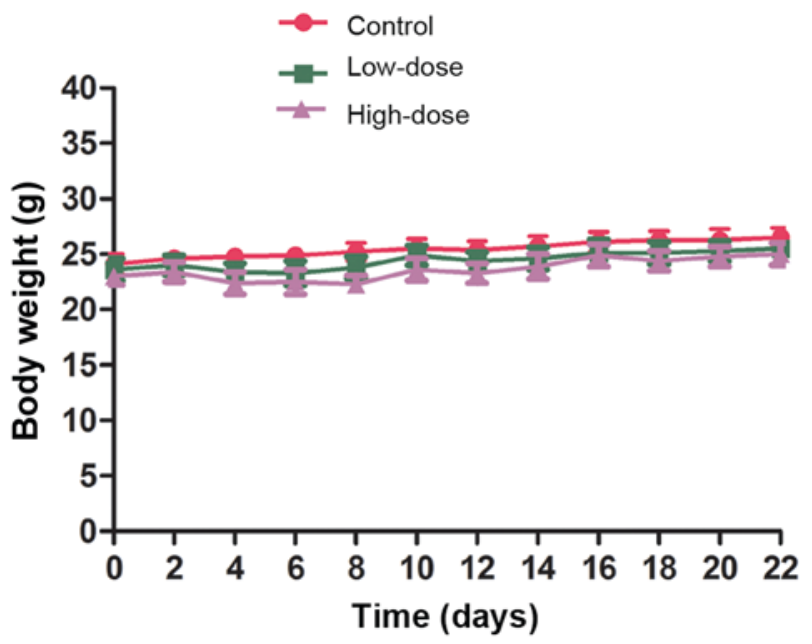

Figure 7. Body weights of mice presented as the mean \pm standard deviation of 5 mice in each group. There were no significant differences between any groups.

(W), taken every other day. Tumor volume was calculated using the following formula: Volume $=(\pi / 6) \times\left(\mathrm{L} \mathrm{x} \mathrm{W}^{2}\right)$. The mice were weighed daily for the 21-day treatment period. After 21 days, the mice were sacrificed and tumor, liver and kidney tissues, as well as blood, were collected for further experimentation. Blood cell counts were analyzed using animal blood analyzers (HEMAVET Veterinary Multi-species Hematology System; Drew Scientific Inc., Miami Lakes, FL, USA). 
A

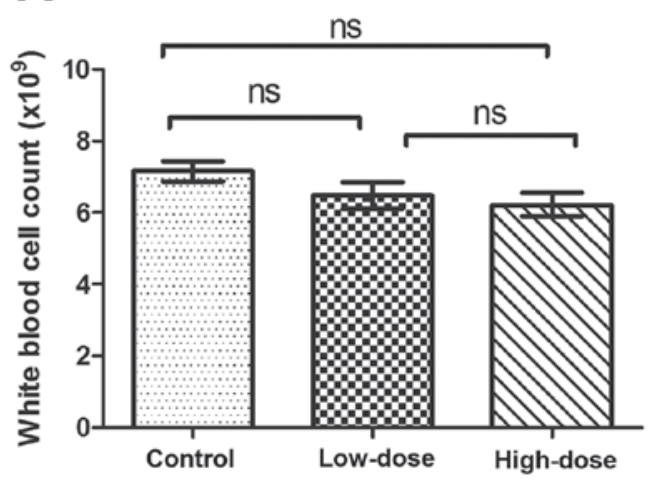

B

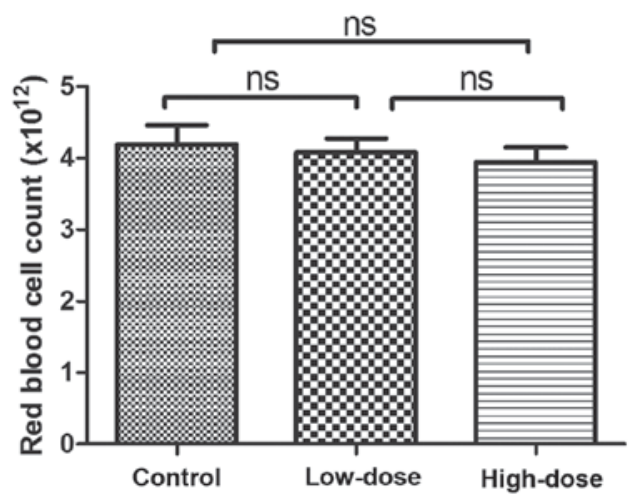

Figure 8. White blood cell and red blood cell counts. (A) White blood cell count in each group, and (B) red blood cell count in each group. There were no significant differences between any groups. ns, not significant.

Statistical analysis. All data are expressed as the mean \pm standard deviation. Statistical analysis was performed using GraphPad Prism (version 5.0; GraphPad Software, Inc., La Jolla, CA, USA). Comparisons between groups were analyzed using Duncan's multiple range test, The correlation analysis was conducted using simple linear regression analysis. $\mathrm{P}<0.05$ was considered to indicate a statistically significant difference.

\section{Results}

LLL12 inhibits the proliferation of human lung cancer cells. The effect of LLL12 on A549-cell proliferation was assessed by MTT assay. This revealed dose- and time-dependent inhibition of cell proliferation with LLL12 treatment (Fig. 1). The $\mathrm{IC}_{50}$ value at $72 \mathrm{~h}$ was $5.0 \mu \mathrm{mol} / \mathrm{l}$, and this concentration was selected for use in subsequent experiments.

LLL12 inhibits the migration of human lung cancer cells. Migration was analyzed using a wound-healing assay and a Transwell migration assay. Following treatment with LLL12, the wound width was compared with that of the control group, and was found to be larger (Fig. 2). In the Transwell assay, the cell number in the bottom compartment was lower in the group treated with LLL12 compared with that in the control group (Fig. 3). These results suggest that LLL12 could inhibit cell migration in human lung cancer cells.

LLL12 inhibits STAT3 phosphorylation in human lung cancer cells. Western blot analysis demonstrated that LLL12 treatment reduced the protein expression level of p-STAT3 and STAT3 significantly compared with that of the control group. However, there was no significant difference in the protein expression level of p-Src or p-ERK1/2 between groups (Fig. 4). This suggests that LLL12 specifically affects STAT3.

LLL12 suppresses tumor growth in vivo. The antitumor effect of LLL12 in vivo was analyzed using a xenograft mouse model. Lung tumor growth was reduced in the groups treated with LLL12 compared with that in the control group. The final tumor volume was significantly lower in the high-dose group compared with that in the low-dose group (Figs. 5 and 6).
Fig. 6A represents the largest tumor diameter obtained. There was no significant difference in the body weight of the mice among any of the groups (Fig. 7).

Safety assessment of LLL12 treatment. During the 21 days of LLL12 administration, mice consumed food and water as normal without vomiting, diarrhea or a significant reduction in body weight. There was no significant difference in white or red blood cell number between groups (Fig. 8).

\section{Discussion}

STAT3 is an important member of the JAK-STAT signaling pathway, and is activated by phosphorylation of a single tyrosine residue located at position 705. STAT3 is phosphorylated when a cytokine or growth factor activates JAK (22). STAT3 has been reported to regulate tumor cell proliferation, differentiation and apoptosis, as well as angiogenesis $(5,19,23)$. Studies have demonstrated that abnormal activation of STAT3 is closely associated with the development of multiple types of cancer, as well as the prognosis of patients with these types of cancer, including head and neck squamous cell carcinoma and prostate cancer $(15,24)$. Morikawa et al $(25)$ suggested that the activation and high expression of p-STAT3 in colorectal cancer may be associated with adverse clinical outcome, indicating its potential role as a prognostic biomarker or therapeutic target. It has also been suggested that cancer-initiating cells may be more sensitive to STAT3 inhibitors (26). Furthermore, it has been reported that the expression of p-STAT3 correlates with tumor differentiation, TNM stage and survival time in gastric cancer, suggesting that STAT3 is a prognostic factor for the disease (27). This is supported by other studies in which the expression levels of STAT3 and p-STAT3 were demonstrated to be closely associated with the progression of gastric cancer, osteosarcoma and esophageal cancer (27-29).

In vitro studies have demonstrated that LLL12 can inhibit the proliferation and induce the apoptosis of various types of cancer cells $(19,20,30,31)$. LLL12 has also been reported to inhibit the formation of tumor blood vessels by downregulating VEGF, matrix metallopeptidase 9 and fibroblast growth factor 1 expression (23). LLL12 has been demonstrated to induce the apoptosis of HCC cells in vitro and to 
induce cell cycle arrest at the G2/M phase (21). It was also reported that LLL12 inhibited liver cancer growth in a mouse model (21). Furthermore, LLL12 has been demonstrated to inhibit the proliferation and migration of osteosarcoma cells in vivo and in vitro $(32,33)$.

In the present study, the in vitro results indicated that LLL12 could inhibit the proliferation of A549 cells in a dose- and time-dependent manner. Metastasis is an important feature of malignant tumors, and a better understanding of the effect of LLL12 on metastasis would be valuable for future research and development of the inhibitor. In the wound-healing assays, the wound width remained wider after $24 \mathrm{~h}$ in cells treated with LLL12 compared with the control. The Transwell assay supported this result by demonstrating that cell migration was decreased following treatment with LLL12 compared with the control. These results suggest that LLL12 could inhibit the migration of human lung cancer cells.

The present study aimed to elucidate the mechanism of LLL12 activity in lung cancer. The results demonstrated that the protein expression levels of STAT3 and p-STAT3 were significantly reduced in cells treated with LLL12 compared with those in the control. However, there was no significant difference in the protein expression level of $\mathrm{p}$-Src or $\mathrm{p}$-ERK1/2 between any groups. These results suggest that LLL12 downregulates the protein expression level of STAT3 and its phosphorylation, which may result in inhibition of proliferation in A549 cells.

The tumor volume in mice treated with LLL12 was significantly reduced compared with that of the control group. Furthermore, tumor volume in the high-dose group was significantly reduced compared with that in the low-dose group. There was no difference in body weight between any groups, and no toxicity was observed. The in vivo study revealed that LLL12 could inhibit cancer tumor growth in a dose-dependent manner. The significant antitumor effect of LLL12 in mice may be associated with downregulated STAT3 expression and phosphorylation. The present study indicates that further studies should focus on the clinical development of LLL12 for the treatment of lung cancer.

\section{Acknowledgements}

The authors would like to thank Professor Tom Li (Ohio State University, Columbus, Ohio, USA) for providing LLL12.

\section{Funding}

This work was supported by funding from the Focus on the Plan Projects of Wuhan Science and Technology Bureau (grant no. 201161038347).

\section{Availability of data and materials}

The datasets used or analyzed during the current study are available from the corresponding author on reasonable request.

\section{Authors' contributions}

SH and YN conceived and designed the study. YN and YL performed the experiments. $\mathrm{YN}$ wrote the paper and $\mathrm{SH}$ reviewed and edited the manuscript. All authors read and approved the final manuscript.

\section{Ethics approval and consent to participate}

The present study was approved by the Ethics Committee of Hubei Cancer Hospital (Wuhan, China).

\section{Patient consent for publication}

Not applicable.

\section{Competing interests}

The authors declare that they have no competing interests.

\section{References}

1. Jemal A, Bray F, Center MM, Ferlay J, Ward E and Forman D: Global cancer statistics. CA Cancer J Clin 61: 69-90, 2011.

2. Pao W and Girard N: New driver mutations in non-small-cell lung cancer. Lancet Oncol 12: 175-180, 2011.

3. Reka AK, Goswami MT, Krishnapuram R, Standiford TJ and Keshamouni VG: Molecular cross-regulation between PPAR- $\gamma$ and other signaling pathways: Implications for lung cancer therapy. Lung Cancer 72: 154-159, 2011.

4. Haura EB, Turkson J and Jove R: Mechanisms of disease: Insights into the emerging role of signal transducers and activators of transcription in cancer. Nat Clin Pract Oncol 2: 315-324, 2005.

5. Kamran MZ, Patil P and Gude RP: Role of STAT3 in cancer metastasis and translational advances. BioMed Res Int 2013: 421821, 2013.

6. Jatiani SS, Baker SJ, Silverman LR and Reddy EP: Jak/STAT pathways in cytokine signaling and myeloproliferative disorders: Approaches for targeted therapies. Genes Cancer 1: 979-993, 2010.

7. $\mathrm{Yu} \mathrm{H}$ and Jove R: The STATs of cancer--new molecular targets come of age. Nat Rev Cancer 4: 97-105, 2004.

8. Looyenga BD, Hutchings D, Cherni I, Kingsley C, Weiss GJ and Mackeigan JP: STAT3 is activated by JAK2 independent of key oncogenic driver mutations in non-small cell lung carcinoma. PloS One 7: e30820, 2012.

9. Xie TX, Wei D, Liu M, Gao AC, Ali-Osman F, Sawaya R and Huang S: Stat3 activation regulates the expression of matrix metalloproteinase-2 and tumor invasion and metastasis. Oncogene 23: 3550-3560, 2004.

10. Yu H, Pardoll D and Jove R: STATs in cancer inflammation and immunity: A leading role for STAT3. Nat Rev Cancer 9: 798-809, 2009.

11. Carpenter RL and Lo HW: STAT3 target genes relevant to human cancers. Cancers (Basel) 6: 897-925, 2014.

12. Debnath B, Xu S and Neamati N: Small molecule inhibitors of signal transducer and activator of transcription 3 (Stat3) protein. J Med Chem 55: 6645-6668, 2012.

13. Masciocchi D, Gelain A, Villa S, Meneghetti F and Barlocco D: Signal transducer and activator of transcription 3 (STAT3): A promising target for anticancer therapy. Future Med Chem 3: 567-597, 2011.

14. Hedvat M, Huszar D, Herrmann A, Gozgit JM, Schroeder A, Sheehy A, Buettner R, Proia D, Kowolik CM, Xin H, et al: The JAK2 inhibitor AZD1480 potently blocks Stat3 signaling and oncogenesis in solid tumors. Cancer Cell 16: 487-497, 2009.

15. Sen M, Thomas SM, Kim S, Yeh JI, Ferris RL, Johnson JT, Duvvuri U, Lee J, Sahu N, Joyce S, et al: First-in-human trial of a STAT3 decoy oligonucleotide in head and neck tumors: Implications for cancer therapy. Cancer Discov 2: 694-705, 2012.

16. Alas S and Bonavida B: Inhibition of constitutive STAT3 activity sensitizes resistant non-Hodgkin's lymphoma and multiple myeloma to chemotherapeutic drug-mediated apoptosis. Clin Cancer Res 9: 316-326, 2003.

17. Sledz CA and Williams BR: RNA interference in biology and disease. Blood 106: 787-794, 2005.

18. Stein CA: The experimental use of antisense oligonucleotides: A guide for the perplexed. J Clin Invest 108: 641-644, 2001. 
19. Lin L, Hutzen B, Li PK, Ball S, Zuo M, DeAngelis S, Foust E, Sobo M, Friedman L, Bhasin D, et al: A novel small molecule, LLL12, inhibits STAT3 phosphorylation and activities and exhibits potent growth-suppressive activity in human cancer cells. Neoplasia 12: 39-50, 2010.

20. Liu A, Liu Y, Li PK, Li C and Lin J: LLL12 inhibits endogenous and exogenous interleukin-6-induced STAT3 phosphorylation in human pancreatic cancer cells. Anticancer Res 31: 2029-2035, 2011

21. Zuo M, Li C, Lin J and Javle M: LLL12, a novel small inhibitor targeting STAT3 for hepatocellular carcinoma therapy. Oncotarget 6: 10940-10949, 2015.

22. Mankan AK and Greten FR: Inhibiting signal transducer and activator of transcription 3: Rationality and rationale design of inhibitors. Exp Opin Investig Drugs 20: 1263-1275, 2011.

23. Bid HK, Oswald D, Li C, London CA, Lin J and Houghton PJ: Anti-angiogenic activity of a small molecule STAT3 inhibitor LLL12. PloS One 7: e35513, 2012.

24. Bishop JL, Thaper D and Zoubeidi A: The multifaceted roles of STAT3 signaling in the progression of prostate cancer. Cancers (Basel) 6: 829-859, 2014.

25. Morikawa T, Baba Y, Yamauchi M, Kuchiba A, Nosho K, Shima K, Tanaka N, Huttenhower C, Frank DA, Fuchs CS and Ogino S: STAT3 expression, molecular features, inflammation patterns, and prognosis in a database of 724 colorectal cancers. Clin Cancer Res 17: 1452-1462, 2011.

26. Lin L, Liu A, Peng Z, Lin HJ, Li PK, Li C and Lin J: STAT3 is necessary for proliferation and survival in colon cancer-initiating cells. Cancer Res 71: 7226-7237, 2011.

27. Xiong H, Du W, Wang JL, Wang YC, Tang JT, Hong J and Fang JY: Constitutive activation of STAT3 is predictive of poor prognosis in human gastric cancer. J Mol Med(Berl) 90: 1037-1046, 2012.
28. Ryu K, Choy E, Yang C, Susa M, Hornicek FJ, Mankin H and Duan Z: Activation of signal transducer and activator of transcription 3 (Stat3) pathway in osteosarcoma cells and overexpression of phosphorylated-Stat 3 correlates with poor prognosis. J Orthopaed Res 28: 971-978, 2010.

29. Schoppmann SF, Jesch B, Friedrich J, Jomrich G, Maroske F and Birner P: Phosphorylation of signal transducer and activator of transcription 3 (STAT3) correlates with Her-2 status, carbonic anhydrase 9 expression and prognosis in esophageal cancer. Clin Exp Metastasis 29: 615-624, 2012.

30. Ball S, Li C, Li PK and Lin J: The small molecule, LLL12, inhibits STAT3 phosphorylation and induces apoptosis in medulloblastoma and glioblastoma cells. PloS One 6: e18820, 2011.

31. Lin L, Benson DM Jr, DeAngelis S, Bakan CE, Li PK, Li C and Lin J: A small molecule, LLL12 inhibits constitutive STAT3 and IL-6-induced STAT3 signaling and exhibits potent growth suppressive activity in human multiple myeloma cells. Int J Cancer 130: 1459-1469, 2012.

32. Onimoe GI, Liu A, Lin L, Wei CC, Schwartz EB, Bhasin D, Li C, Fuchs JR, Li PK, Houghton P, et al: Small molecules, LLL12 and FLLL32, inhibit STAT3 and exhibit potent growth suppressive activity in osteosarcoma cells and tumor growth in mice. Invest New Drugs 30: 916-926, 2012.

33. Couto JI, Bear MD, Lin J, Pennel M, Kulp SK, Kisseberth WC and London CA: Biologic activity of the novel small molecule STAT3 inhibitor LLL12 against canine osteosarcoma cell lines. BMC Vet Res 8: 244, 2012. 